\title{
Design, development and optimization of topotecan hydrochloride solid lipid nanoparticles for oral chemotherapy
}

\begin{abstract}
The objective of this research work was to formulate and evaluate solid lipid nanoparticles of anticancer drug topotecan hydrochloride in order to sustain its release and therefore improve its therapeutic outcomes. SLNs were prepared by hot micro emulsion method using glyceryl monostearate as lipid carrier and Tween 80 as surfactant in specific proportions. FT-IR and DSC studies revealed that there was no interaction between drug and lipid. The effect of drug: lipid ratio, surfactant concentration, speed and time of homogenizer on drug release, particle size and EE were studied. The results of particle size, encapsulation efficiency and in vitro drug release at $12 \mathrm{hr}$ fell between $111.9 \mathrm{~nm}$ and $255 \mathrm{~nm}, 65.12 \%$ and $77.63 \%$, and 72.31 and $79.79 \%$ respectively. Optimized formulation showed release of drug up to $73.47 \%$ at $12 \mathrm{hrs}$, entrapment efficiency of $77.75 \%$ and particle size of $158.0 \mathrm{~nm}$. The analysis of 3-D graphs revealed that parameters such as drug: lipid ratio and time of homogenization have a profound effect on both encapsulation efficiency and cumulative drug release. Scanning electron microscopy studies showed particles with disuniform surface and shape. The drug release from optimized formulation was found to fit best into first order kinetic model. It also showed almost linear regression in Higuchi's plot suggesting that diffusion is one of the mechanisms for drug release and $\mathrm{n}$ value of Korsmeyer-Peppas plot was found to be 0.716 indicating that the drug release did not follow fickian diffusion controlled mechanism.
\end{abstract}

Keywords: Solid lipid nanoparticles, Glyceryl monostearate, Surfactant, Encapsulation efficiency, In vitro release studies
Volume 3 Issue I - 2016

\author{
llangala Booka A,' Prakash Rao B,' Ciza \\ Hamuli P,' Rajanrajan ${ }^{2}$ \\ 'Department of Pharmaceutical Technology, Rajiv Gandhi \\ University of Health Sciences, India \\ ${ }^{2}$ Department of Pharmaceutics, Karnataka College of Pharmacy, \\ India
}

\begin{abstract}
Correspondence: Booka Ange, Research scholar, Department of Pharmaceutical Technology, Rajiv Gandhi University of health Sciences, \#33/I,Thirumenahalli, Jakkur post, yelahanka Hobli, Bangalore, 560064, India, Tel 917259000000 Email angeilangala@yahoo.fr, angeilangalal4@gmail.com
\end{abstract}

Received: December 26, 2019 | Published: January 08, 2016
Abbreviations: SLN, Solid Lipid Nanoparticles; DLS, Dynamic Light Scattering; PdI, Polydispersity Index; FT-IR, Fourier Transform Infrared Spectroscopy; EE, Entrapment Efficiency

\section{Introduction}

Solid lipid nanoparticles (SLN) firstly introduced in 1991 by Muller and Gasco, represent an alternative carrier system to traditional colloidal carriers such as emulsions, liposomes and polymeric micro/ nanoparticles.

SLNs are sub-micron colloidal carriers ranging from 50 to 1000 $\mathrm{nm}$, which are composed of physiological lipid, dispersed in water or in aqueous surfactant solution. ${ }^{2,3}$ They offer unique properties such as small size, large surface area, high drug loading and are attractive for their potential to improve performance of pharmaceuticals.4-6 Furthermore, they also have the ability to encapsulate both hydrophobic and hydrophilic drugs, improving thus their physico-chemical and pharmacokinetics profile. ${ }^{1,5}$ Solid lipid nanoparticles have many advantages namely low cost of ingredients, ease of preparation and scale-up, high drug payload, no biotoxicity of the carrier, possibility of controlled drug release and targeting. 5,6

There are various method of preparation for SLNs, each one providing special features, advantages and limitations. These methods include High pressure homogenization, ultra sonication Techniques, Solvent evaporation method, solvent ejection method (solvent displacement method), Solvent emulsification-diffusion method, supercritical fluid method, micro emulsion based method, spray drying method, Double emulsion method and Precipitation technique. ${ }^{7-11}$
Oral chemotherapy is a key step towards chemotherapy at home, a dream of cancer patients, which will radically change the clinical practice of chemotherapy and greatly improve the quality of life of the patients. ${ }^{12}$ However, this route sounds very challenging due to the low aqueous solubility, poor intestinal permeability, high level of P-glycoprotein (P-gp) efflux and pre-systemic metabolism of most of anticancer drugs being currently used. Many research works have showed that nanotechnology based drug delivery have the ability to encapsulate various anticancer drugs, offering thus a valuable mean to overcome the above issues. ${ }^{13-15}$ Topotecan (9-[(dimethyl amino) methyl]-10-hydroxy- (20S) - camptothecin hydrochloride) is one of the two camptothecin analogues currently approved for clinical application by FDA for the treatment of refractory ovarian cancer and/or small-cell lung cancer, which are resistant to conventional chemotherapeutic agents. In addition, the combination of topotecan with other standard chemotherapeutic agents is progressively being used for optimized therapy. ${ }^{16,17}$ Nowadays, there are considerable data in the literature addressing the toxicity level of topotecan. Some studies showed topotecan as being relatively well tolerated at low dose basis with non hematologic toxicities that include nausea, vomiting, and dyspnea and these side effects are generally mild to moderate in severity. ${ }^{18,19}$ Nevertheless, when the standard dosing regimen is considered (Topotecan $1.5 \mathrm{mg} / \mathrm{m} 2$ on days 1 to 5 of a 21 -day cycle), the drug has been associated with increased bone marrow toxicity and severe haematological toxicities, limiting the practicality of using the drug in patients with low performance status. ${ }^{20}$

Preclinical studies demonstrated greater antitumor efficacy with prolonged low-dose exposure to topotecan, and in animal models, low-dose prolonged exposure resulted in less toxicity. Therefore, 
increased antitumor efficacy and decreased toxicity could be achieved by prolonged exposure of tumour tissues to the drug. ${ }^{21,22}$ Many studies have reported the encapsulation of Topotecan within liposomes, but there are barely any reports on preparation of topotecan loaded solid lipid nanoparticles as an attempt to sustain or prolong its release following oral administration. Hot micro emulsion based method is a highly accessible method which does not require sophisticated equipment to produce solid lipid nanoparticles. Hence, the overall aim of this work was to formulate and evaluate topotecan hydrochloride loaded solid lipid nanoparticles in order to achieve sustained release profile and therefore improved efficacy of topotecan and decrease the toxicity level of this drug as well.

\section{Materials and methods}

\section{Materials}

Topotecan hydrochloride was gifted sample of Strides arcolab limited, Bangalore, India. Glycerol monostearate was purchased from Aka fine chemicals, Bangalore, India. Tween 80 was purchased from S.D Fine chemicals, Mumbai, India. Poloxamer 188 (Pluronic F-68) was purchased from HiMedia laboratory, pvt ltd, Mumbai, India. Potassium dihydrogen phosphate was procured from Qualigens Fine Chemicals, Mumbai, India. Sodium hydroxide was obtained from Rolex Chemical Industries, Mumbai, India

\section{Methods}

Fourier Transform Infrared Spectroscopy (FT-IR)

The drug excipients interaction studies were carried out by scanning A) Pure drug B) Drug + Glyceryl monostearate C) Drug + Tween 80 using FT-IR spectrophotometer (Bruker, $\alpha$-E: ATR model, Germany) in the range of $4000-500 \mathrm{~cm}^{-1}$ and any incompatibility associated with the drug and excipients were studied.

\section{Differential scanning calorimetry}

The Differential Scanning Calorimetry was employed as tool to investigate the physico-chemical incompatibility between the drug and commonly used excipients which might affect the stability of the drug by chemical and physical interactions thus posing a threat to the stability or bioavailability. DSC was carried out using a DSC Q200 (TA instruments, US). A mass of $5 \mathrm{mg}$ samples were accurately weighted, sealed in an aluminium pan and equilibrated at 25 C, which were subjected to a heating run over the temperature range of 25-250oC, except for GMS whose we scanned up to $100 \mathrm{oC}$ only.

\section{X- Ray diffraction}

The X-Ray diffraction of Topotecan hydrochloride formulation was done to find out any change in the crystallinity of the drug during microencapsulation by using Siemens DX500. PXRD studies were performed by exposing the samples to $\mathrm{CuK} \alpha$ radiation $(45 \mathrm{kV}, 40 \mathrm{~mA})$ and scanning from $5^{\circ}$ to $50^{\circ}, 2 \theta$ at a step size of $0.017^{\circ}$ and scan step time of $25 \mathrm{~s}$.

\section{Preparation of topotecan loaded SLNs using micro emulsion method}

Solid lipid of topotecan hydrochloride was prepared by hot micro emulsion based method. Briefly, the drug $(10 \mathrm{mg})$ was previously dissolved in methanol and dispersed in melted lipid (glyceryl monostearate), and then the mixture was dispersed in a hot aqueous solution with surfactant(tween 80 ) concentration ranging from $1 \%$ to $2 \%$, by high - speed stirring, using an Ultra-Turrax homogenizer at specific rpm for an appropriate period of time. The resulting dispersion was then cooled and each sample was diluted with water before the particle size was measured using dynamic laser light scattering apparatus at $25^{\circ} \mathrm{C}$.

\section{Mean particle size, polydispersity index ( $\mathrm{PdI})$ and zeta potential}

Dynamic light scattering (DLS) was used to assess the mean particle size and PdI of the solid lipid nanoparticles dispersions. Samples were diluted (1:50) with distilled water and analysed using a Zetasizer Nano S (Malvern Instruments, UK). Zeta potential was determined from electrophoretic mobility using a Zetasizer Nano ZS (Malvern Instruments, UK).

\section{Percentage yield}

To obtain the percentage yield, the obtained solid lipid nanoparticles were lyophilised and then weighted. \% yield was calculated by using following formula.

\section{Determination of drug content}

The amount of SLNs equivalent to $5 \mathrm{mg}$ Topotecan hydrochloride were accurately weighed and then added to $100 \mathrm{ml}$ of methanol. The samples were heated at $65^{\circ} \mathrm{C}$ to melt the carrier. Heating was done for $20 \mathrm{~min}$. The solutions were centrifuged at $20000 \mathrm{rpm}$ for $30 \mathrm{~min}$. The supernatant was collected and the drug content was estimated by UV Spectrophotometer at $382 \mathrm{~nm}$.

The \% drug content in the SLM was calculated by using the following equation

\section{Determination of Entrapment efficiency (EE)}

SLNs equivalent to $50 \mathrm{mg}$ of topotecan hydrochloride were accurately weighed and then added to $100 \mathrm{ml}$ of methanol. The solutions were kept shaking for $1 \mathrm{hr}$. Then the solutions were centrifuged at $20000 \mathrm{rpm}$ for $30 \mathrm{~min}$. The supernatant was collected and the drug content was estimated by UV Spectrophotometer at 382 $\mathrm{nm}^{20}$

Where;

$\mathrm{W}_{\mathrm{i}}$ is the amount of drug added to SLNs

$\mathrm{W}_{\mathrm{f}}$ is the amount of free drug detected in the supernatant.

\section{In vitro diffusion study}

The diffusion study of prepared solid nanoparticles was performed using cellulose dialysis membrane with a molecular weight cut-off of $12,000 \mathrm{Da}$ and phosphate buffer $(\mathrm{pH} 6.8)$ at $370 \mathrm{C} \pm 0.50 \mathrm{C}$. The Franz diffusion cell of $140 \mathrm{ml}$ capacity was used. The composition equivalent to $3.5 \mathrm{mg}$ of topotecan hydrochloride was applied on the membrane and diffusion study was performed over a period of $12 \mathrm{hrs}$. The samples $(3 \mathrm{ml})$ were withdrawn at $1,2,3,4,5,6,7,8$, and 12 hour and fresh medium were added to maintain sink condition. The collected samples were filtered through $0.22 \mu \mathrm{l}$ filter and analysed at $382 \mathrm{~nm}$ by using UV/VIS Spectrophotometer. The obtained data were subjected to release kinetics studies by applying four kinetics models to the data in order to determine the best fitting equations $\mathrm{s}^{23,24}$

Zero order equation: $\mathrm{Q}=\mathrm{Q}_{0}-\mathrm{k}_{0} \mathrm{t}$
First order equation: $\ln =\ln \mathrm{Q}-\mathrm{k} 1 \mathrm{t}$
Higuchi equation: $\mathrm{Q}=\mathrm{k}_{2} \mathrm{t}^{1 / 2}$
Korsmeyer peppas equation: $\mathrm{Q} / \mathrm{Q}_{0}=\mathrm{kt}^{\mathrm{n}}$
$\mathrm{K}_{0}$ to $\mathrm{K}_{2}$ were release rate constant, $\mathrm{Q} / \mathrm{Q}_{0}$ was fraction of drug 
released at time $\mathrm{t}, \mathrm{K}$ was constant $\mathrm{n}$ was diffusion constant that indicates general operating release mechanism.

\section{Optimization}

The formulations were designed based on Taguchi OA design and then evaluated for the response. Four input factors were studied at three levels $\left(3^{4}\right)$ throughout the preparation process to determine their effect on three responses, namely mean particle size, encapsulation efficiency and drug at $1 \mathrm{hr}, 8 \mathrm{hr}$ and $12 \mathrm{hr}$. The input factors being selected are the following: Drug to lipid ratio $(1 / 20,10 / 250$, and 10/300), concentration of surfactant $(1 \%, 1.5 \%, 2 \%)$ speed (14000RPM, 16000RPM, 18000 RPM) and time of homogenization (5min, $10 \mathrm{~min}$ and $15 \mathrm{~min}$ ). The response values were subjected to multiple regression analysis to find out the relationship between the input factors used and the response values obtained. Therefore, the objective of the optimization process was to quantify the effect of the above factors on the solid lipid nanoparticles. The multiple regression analysis was done using design expert 9.0.4 software, which is specially meant for this optimization process.

In the numerical optimization techniques, the desirability approach was used to generate the optimum settings for the formulation. For the optimized formulation, the particles size was kept at the targeted value, the drug release at $1^{\text {st }} \mathrm{hr}$ was kept at minimum, the drug release at $8^{\text {th }} \mathrm{hr}$ was kept at minimum, and drug release at $12^{\text {th }} \mathrm{hr}$ was kept at minimum. The encapsulation efficiency was also kept at maximum. The optimized formulation was prepared according to predicted model and evaluated for responses. The result of the optimized formulation has been further compared with the predicted model values.

\section{Scanning electron microscope}

The solid sample was sputter-coated using a thin gold-palladium layer under an argon atmosphere using a gold sputter module in a highvacuum evaporator (JFC-1100 fine coat ion sputter; Tokyo, Japan). These coated samples were then scanned and photomicrographs were taken at an acceleration voltage of $5 \mathrm{kV}$.

\section{Statistical analysis}

Data were analysed with the help of one-way analysis of variance (ANOVA) test and parameters were significant for the $\mathrm{p}<0.05$.

\section{Results and discussion}

Fourier Transform Infra-red (FT-IR) studies.

The spectrum obtained from FT infrared spectroscopy studies at wavelength from $4000 \mathrm{~cm}^{-1}-500 \mathrm{~cm}^{-1}$ are shown in Figure 1 Characteristic peaks in the region of $1732.90 \mathrm{~cm}^{-1}, 1468.06 \mathrm{~cm}^{-1}$, $3308.54 \mathrm{~cm}^{-1}, 1111.22 \mathrm{~cm}^{-1}, 1457.83 \mathrm{~cm}^{-1}$ were found to be observed in physical mixtures which were identical to that of the pure drug; this confirmed the intactness of the drug in the physical mixture.

Differential Scanning Calorimetry (DSC) studies and XRD studies.

In DSC studies melting peak appeared at $191^{\circ} \mathrm{C}$ for topotecan hydrochloride and $53.04^{\circ} \mathrm{C}$ for glyceryl monostearate as showed in Figure 2 Slight shift in peak position for both topotecan and glyceryl monostearate was observed in physical mixture indicating that there is no interaction between topotecan and lipid. Seemingly, the absence of Topotecan endotherm peak in thermo grams of SLN formulation argues that majority of Topotecan was present in amorphous form within the lipid matrix. After SLNs formation, reduction in crystallinity (reduced number and intensity of peaks) was observed in PXRD spectrum for the drug. This simply proves presence of molecular level dispersion and therefore the efficiency of the dispersion of the drug within the lipid carrier after SLN formation and also strengthens the hypothesis that crystallinity of Topotecan gets reduced significantly in SLN formation. PXRD data are presented in Figure 3.

\section{Evaluation and characterization of Topotecan SLNs}

Particles size, \% drug content and encapsulation efficiency The prepared formulations have been analysed for drug content, encapsulation efficiency and mean particles size, and polydispersity index. Under the optimum preparation conditions, small lipid nanoparticles with a relatively uniform size distribution were obtained. Figure 4 The mean dynamic light scattering particle diameters fell between $111.9 \mathrm{~nm}$ and $255 \mathrm{~nm}$ with PDI values below 0.3 indicating fair size distributions. ${ }^{25,26}$ Encapsulation of water soluble or hydrophilic drug into a solid lipid matrix is one of the challenges associated with this type of formulation and requires appropriate approach to address $\mathrm{it}^{27}$ So far, the optimized formulation has shown up to $81.75 \%$ encapsulation efficiency. The relatively high level of encapsulation plays an important role in the achievement of desirable prolonged release effect. Thus, it can be concluded that hot micro emulsion method and excipients used in this study are suitable to produce TPT-SLNs. Table 1 Presents the results of particles size, $\%$ drug content and encapsulation efficiency of all the formulations.

Table I Mean diameter, polydispersity index (PDI), entrapment efficiency (EE), and drug content (DC)

\begin{tabular}{lllll}
\hline Sample series & $\begin{array}{l}\text { Mean diameter } \\
\text { (In Nm) }\end{array}$ & PDI & EE (\%W/W) & DC (\%W/W) \\
\hline FI & I80 & 0.366 & 69.25 & 96.86 \\
F2 & I5I & 0.353 & 71.24 & 93.21 \\
F3 & I58 & 0.375 & 70.44 & 90.12 \\
F4 & 255.5 & 0.296 & 74.44 & 95.43 \\
F5 & 213.3 & 0.337 & 65.12 & 94.32 \\
F6 & 143.5 & 0.351 & 73.24 & 97.35 \\
F7 & 137.5 & 0.314 & 77.63 & 92.13 \\
F8 & 114.4 & 0.341 & 68.85 & 90.67 \\
F9 & III.9 & 0.287 & 67.92 & 91.74 \\
FI0 (optimized) 158 & 0.301 & 81.75 & 97.82
\end{tabular}

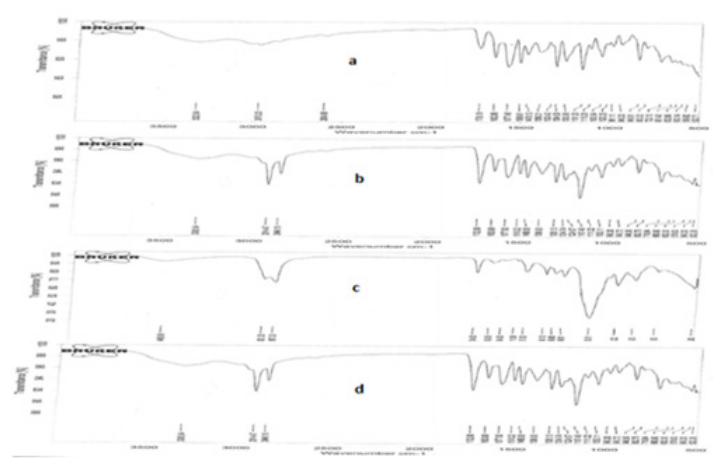

Figure I FTIR spectra of TPT (A), Physical mixture of TPT+GMS (B), physical mixture of TPT+Tween $80(\mathrm{C})$ and physical mixture of TPT + Pluronic F-68 (D).

\section{In-vitro drug release studies and drug release kinetics}

The in vitro release of Topotecan hydrochloride from the prepared solid lipid nanoparticle formulations and pure drug was studied in $\mathrm{pH} 6.8$ phosphate buffer as that is the intestinal $\mathrm{pH}$. The drug release was measured for $12 \mathrm{hr}$. The pure drug has showed complete release at around 3 hours. This is not surprising since the drug is not in a sustained formulation. In contrary, for the TPT-SLN formulation, prolonged release was noticed, which suggests that the drug was 
significantly encapsulated insight the lipid matrix. ${ }^{28}$ The cumulative drug release for the optimized formulation was about $73.47 \%$ at 12 hr Figure 5.

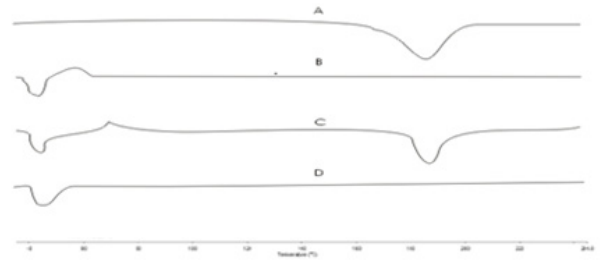

Figure 2 DSC thermo grams of TPT (A), GMS (B), physical mixture of TP and GMS (C) and TPT-SLNs (D).
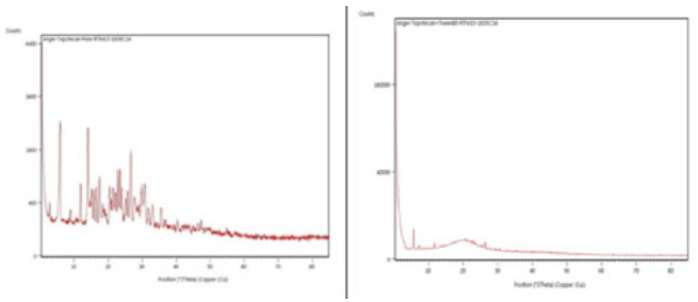

Figure 3 XRD of topotecan pure drug (left) andTPT-SLNs formulation (right).

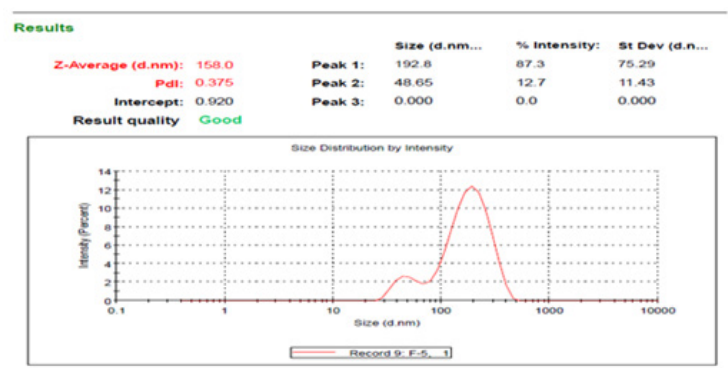

Figure 4 Dynamic light scattering mean particles size of TPT-SLNS optimized formulation.

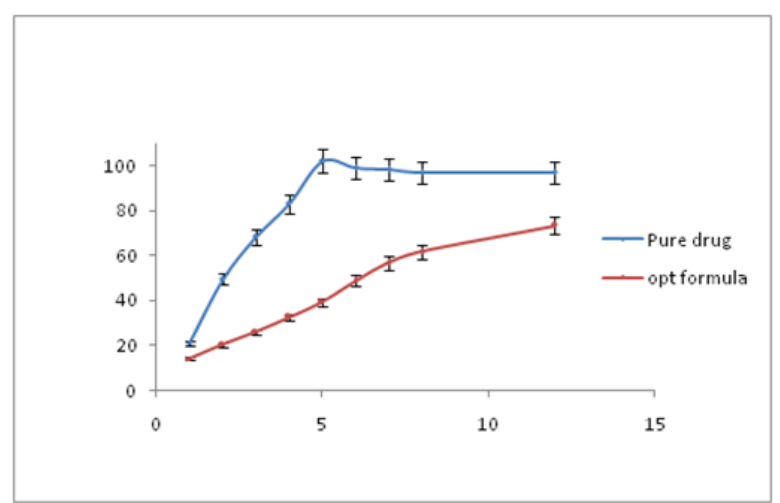

Figure 5 In vitro release data of TPT-SLNs formulation and TPT pure drug.

Drug release mechanisms and kinetics are the two important characteristics of a delivery system in describing the drug dissolution or diffusion profile. It is now well known that qualitative and quantitative changes in the formulation but also in the processing conditions may alter drug release and in vivo performances of a pharmaceutical system. The mathematical models methods represent one the rational approaches to assess and predict the in vivo bioperformance of new delivery systems. In this perspective, the drug release data of TPT-SLN formulations were fitted into different models like zero order, first order, Higuchi equation and Korsmeyer-peppas. The correlation coefficient value (R2) was used as an indicator to find the best fitting equation for the prepared TPT-SLN formulations. The drug release from optimized formulation was found to fit best into first order kinetic model, which means that the drug release is proportional to the amount of drug remaining inside the delivery system. It also showed almost linear regression in Higuchi's plot suggesting that diffusion is one of the mechanisms for drug release and $n$ value of Korsmeyer-Peppas plot was found to be 0.716 indicating that the drug release did not follow fickian diffusion controlled mechanism. The non-Fickian diffusion might suggest that rates of solvent penetration and drug release are in the same range. Based on these results we can conclude that the prepared TPT-SLNs formulation fit for a diffusion matrix formulation. The result of release kinetics studies are presented Figure 6
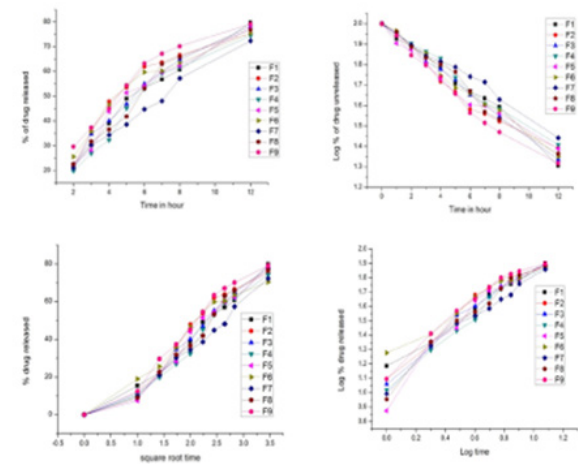

Figure 6 Release kinetics of different TPT-solid lipid nanoparticles formulations.

\section{Effect of formulation and process variables on mean particles size}

Particle size is a critical characteristic of nanoparticles and by definition differentiates them from micro particles. Particle size and particle size distribution play an important role in the biological performance of the nanoparticles. ${ }^{29}$ Nowadays, Nanoparticles are becoming very attractive because of their important and unique features, such as their surface to mass ratio that is much larger than that of other particles, and their ability to adsorb and carry a broad range of medical relevant compounds like drugs and proteins. ${ }^{30}$ Both formulation and process variable might affect the mean particle size and size distribution of solid lipid nanoparticles during the production. Therefore, the effect of drug to lipid ratio and concentration of surfactant on the particle size was studied. The model was found to be significant with an F value of $8.05(\mathrm{p}<0.0339)$. Figure 7 represents the observed response values compared to that of predicted values. From the $3 \mathrm{D}$ graph it was found that $1.5 \%$ of tween 80 concentration gave the smaller particles size. Moreover, the particle size turned to decrease with increase in the speed of stirring and the particle size was found to be increased with increase in the lipid content.
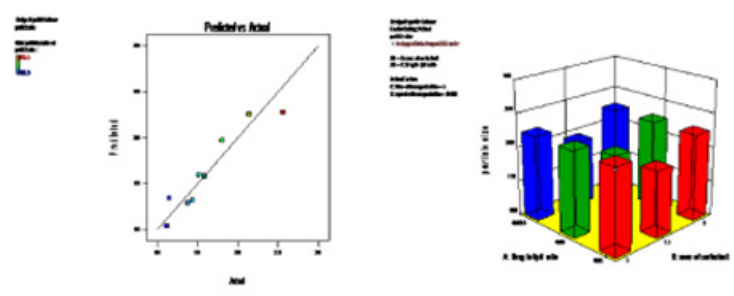

Figure 7 3-D graph showing effect of drug: lipid ratio and Tween 80 conc on mean particle size (RI) 


\section{Effect of formulation and process variables on encapsulation efficiency}

One of the major challenges in the development of nanocarriers based drug delivery is to engineer a system that can effectively encapsulate drugs at high concentration. The encapsulation offers several advantages such as protection, improvement of intrinsic properties of the encapsulated molecules, targeting properties, and sustained release effect. ${ }^{31}$ For this reason, the effect of formulation and process variables on encapsulation efficiency was investigated. The model was found to be significant with an F value of 49.19 ( $p<$ 0.0201 ). Figure 8 represents the observed response values compared to that of predicted values. The 3-D graph shows that the encapsulation efficiency increased with increase of lipid amount and concentration of surfactant. However, the concentration of surfactant affects much less on the $\% \mathrm{EE}$ as compare to the effect of lipid amount.
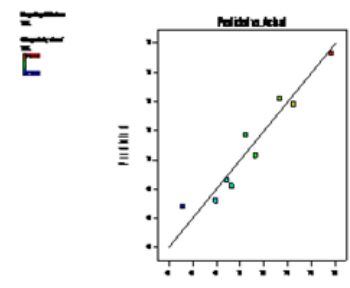

$=$
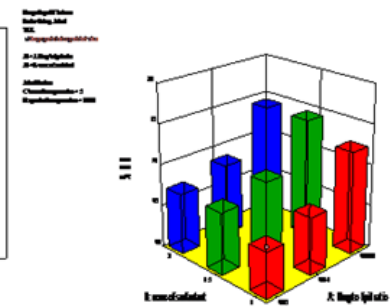

Figure 8 3-D graph showing effect of drug: lipid ratio and Tween 80 conc on encapsulation efficiency (R2)

\section{Effect of formulation and process variables on drug} release profile

There is a considerable number of studies which present solid lipid nanoparticles as tool to achieve sustained or prolong release of encapsulated molecules. In this particular case, a couple of studies have demonstrated the relevance of prolonged exposure of topoisomerase I inhibitors might be beneficial in improving the clinical efficacy of such drug. ${ }^{32,33}$ From this basis, the attempt in this work was to sustain the release of topotecan in order to optimize its therapeutic outcome following oral administration. Therefore, the effect of formulation and process variables on the release pattern was studied. The model was found to be significant with an F value of $6.44(\mathrm{p}<0.0321), \mathrm{F}$ value of $21.50(\mathrm{p}<0.0451)$, and $\mathrm{F}$ value of $5.21(\mathrm{p}<0.0479)$ respectively for release $1 \mathrm{hr}, 8 \mathrm{hr}$, and $12 \mathrm{hr}$. From the 3 -D graphs Figure 9 it can be learned that the increase in the time of homogenization results in a significant raise of $1 \mathrm{hr}$ release. The explanation may be as the time homogenization increases, the particles size goes smaller and therefore increase the rate of drug release. The $8 \mathrm{hr}$ release decrease with an increase of lipid concentration.

The optimize formulation was evaluated and the experimental values were compared to the predicted ones as illustrated in Figure 10. The summary of Anova results is presented in Table 2.

\section{Scanning electron microscope}

The particles size and shape may affect several properties of nanotherapeutics systems such as drug release rate, ability to interact with biological systems etc. Therefore, the surface morphology and size of lyophilized TPT- Solid lipid nanoparticles was studied using scanning electron microscope. The Figure 11 shows particles with quite disuniform surface and shape. The size of the particles from SEM was slightly different in comparison to dynamic light scattering result. This might be due to influence of the lyophilisation process since for SEM analysis we have used lyophilised samples.
Table 2 Summary of Anova results in analysing lack of fit (LOF)

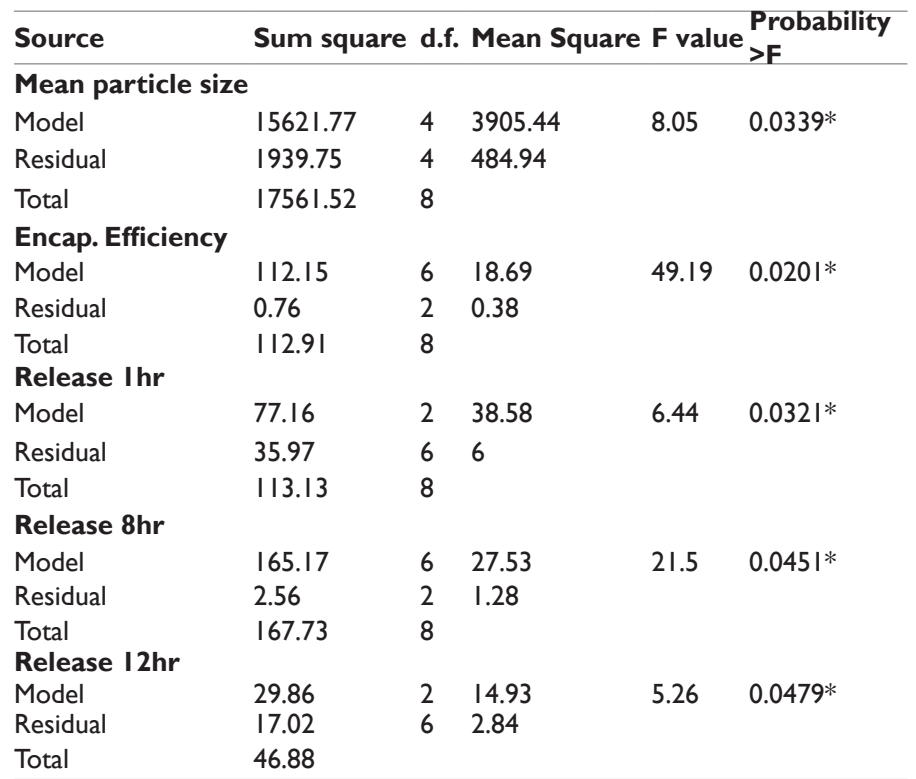
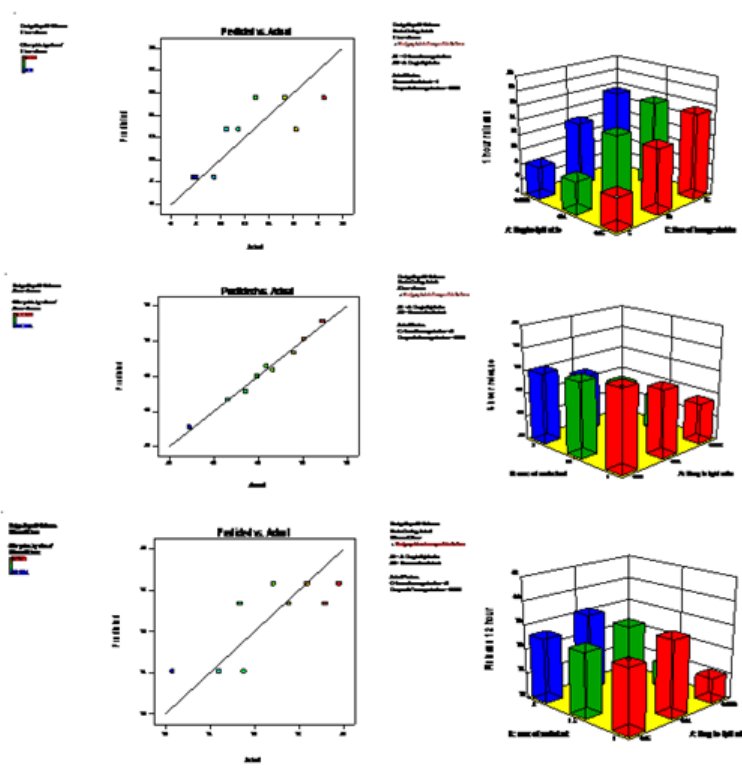

Figure 9 3-D graph showing effect of input variables on drug release $\mathrm{I} \mathrm{hr}$, $8 \mathrm{hr}$ and $12 \mathrm{hr}$.

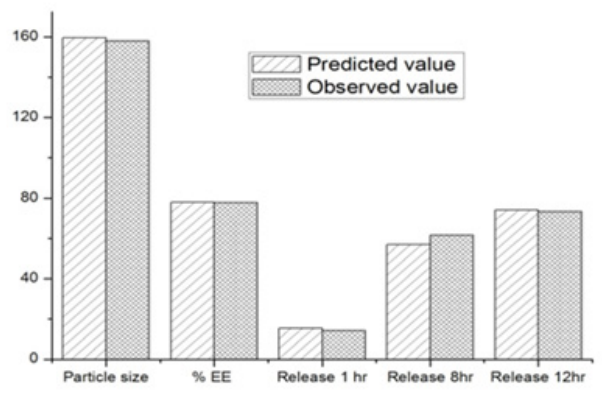

Figure 10 The comparison between predicted and experimental value for Particles size (I), Encapsulation efficiency (2), Release Ihr (3), Release 8hr (4), and Release I $2 \mathrm{hr}$ (5). 


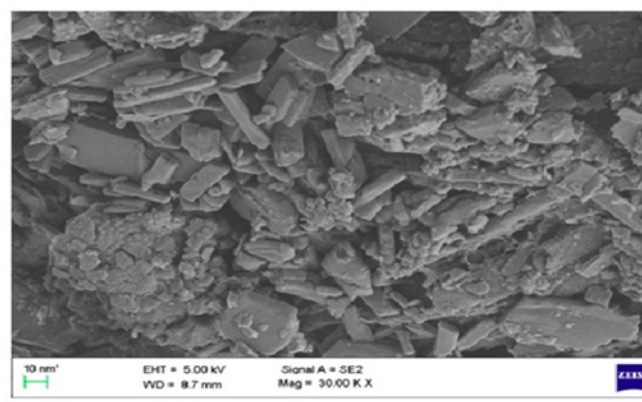

Figure I I SEM of optimized formulation.

\section{Conclusion}

This study has demonstrated the feasibility of the development of topotecan loaded solid lipid nanoparticles using Taguchi design approach. The sustained release property of this formulation as well as the in vitro release kinetics model were illustrated. The analysis of 3-D graphs has provided a deep understanding of the effect of input variables on the selected responses. Therefore, it can be concluded that the method and optimization approach adopted throughout this study are suitable for producing topotecan-SLNs.

\section{Acknowledgements}

We are grateful to Dr. Vashuda from Raman institute, Bangalore for the Differential scanning calorimetry and X-ray diffraction analyses. This work was supported by The Excellence Scholarship Program BEBUC(HYPERLINK "http://www.foerderverein-uni-kinshasa. de/" It "_blank" www.foerderverein-uni- kinshasa.de), by the ElseKroener-Fresenius- Stiftung and the Holger-Poehlmann-Stiftung." (A. Ilangala).

\section{Conflicts of interest}

None.

\section{References}

1. Ekambaram P, Abdul hasan sathali, Priyanka K. Solid lipid nanoparticles: a review. sci. rev. chem. Commun. 2012;2(1):80-102.

2. yadav N, sunil K, sara UDS. Solid lipid nanoparticles- a review. International Journal of Applied Pharmaceutics. 2013;5(2):8-18.

3. Jawahar N, Meyyanathan S.N, Gowtham R, et al. Solid lipid Nanoparticles for Oral delivery of Poorly Soluble Drugs. Journal of Pharmaceutical Science and Research . 2012;4(7):1848-1855.

4. Patricia S, Andreani T, Macedo AS, et.al. Current State-of-Art and New Trends on Lipid Nanoparticles (SLN and NLC) for Oral Drug Delivery. J Drug Deliv ID 750891. 2012;p:10.

5. Maryam G, Shohreh F, Alireza V, et al. Loading hydrophilic drug in solid lipid media as nanoparticles: Statistical modeling of entrapment efficiency and particle size. International Journal of Pharmaceutics. 2012;424(1-2):128-137.

6. Anu M, Sandeep K, Navjit KG, et al. Solid lipid nanoparticles (SLNs) - as novel lipid based nanocarriers for drugs. International Journal of Advanced Research. 2014;2(1):433-441.

7. Wolfgang M, Karsten M. Solid lipid nanoparticles: Production, characterization and applications. Adv Drug Deliv Rev. 2012;47(2-3):165-196

8. Anu M, Sandeep K, Navjit KG, et al. Solid lipid nanoparticles (SLNs) - as novel lipid based nanocarriers for drugs. International Journal of Advanced Research. 2014;2(1):433-441.
9. Neha Yadav, Sunil Khatak, Udai Vir Singh Sara. Solid Lipid Nanoparticles- A Review. International Journal of Applied Pharmaceutics. 2013;5(2):397-410.

10. Jagdevappa P, Prashant G, Ravindra K, et al. Applications of solid lipid nanoparticle in novel drug delivery system. British biomedical bulletin. 2013;1(2):103-118

11. Akanksha g, deepti s, navneet g. Solid lipid nanoparticles (SLN): method, characterization and applications. International Current Pharmaceutical Journal. 2012;1(11):384-393.

12. Lin M, Zhiping Z, Lingyun Z, et al. Pharmaceutical nanotechnology for oral delivery of anticancer drugs. Advanced Drug Delivery Reviews. 2013;65(6):880-890.

13. Silva M, Kawthar B, Gilles P. Oral delivery of anticancer drugs I: general considerations. Drug Discov Today. 2013;18:1-2:25-34.

14. Silva M, Kawthar B, Gilles P. Oral delivery of anticancer drugs III formulation using drug delivery systems. Drug discovery today. 2013;18:1-2:94-104.

15. Kaushik T, RP. Gangwal, Sangamwar AT, et al. Oral delivery of anticancer drugs: challenges and opportumity. J Control Release. 2013;170(1):15-40

16. Dadashzadeh S, Vali M, Rezaie M. The effect of PEG coating on in vitro cytotoxicity and in vivo disposition of topotecan loaded liposomes in rats. Intern. J of Pharm. 2008;353(1-2):251-259.

17. Tahir K, Devina V, Sushama T, et al. Topotecan-tamoxifen duple PLGA polymeric nanoparticles: investigation of in vitro, in vivo and cellular uptake potential. Int J Pharm. 2014;473(1-2):384-394.

18. Yi XF, Fan SM, Yao M, et al. Comparison of efficacy and toxicity profile between intraperitoneal and intravenous topotecan in human ovarian cancer xenografts. Beijing Da Xue Xue Bao, 2006;38(1):88-91.

19. Garst J. An evolving option in the treatment of relapsed small cell lung cancer. Ther Clin Risk Manag. 2007;3(6):1087-1095.

20. Jalid Sehouli. Topotecan weekly versus topotecan five-day schedule in patients with platinium-resistant ovarian cancer-which is the best regimen? European oncological desease. 2007;1(2):84-85.

21.Pantazis P, Kozielski A, Rodriguez R, et al. Therapeutic efficacy of camptothecin derivatives against human malignant melanoma xenografts. Melanoma Research. 1994;4(1):5-10.

22.P.W.C. Akkermans. Topoisomerase I inhibitors: The relevance of prolonged exposure for present, clinical development, in: P.J. B0S (Eds), Prolonged tumor exposure to topoisomerase I inhibitor. 1997;2:32-33.

23. Dash S1, Murthy PN, Nath L, et al. Kinetic modeling on drug release from controlled Drug delivery systems. Acta Pol Pharm. 2010;67(3):217-223.

24. Subhashree S, Chandra KC, Pradipta KB. Development and Evaluation of Gastroretentive Controlled Release Polymeric Suspensions Containing Ciprofloxacin and Carbopol Polymers. J Adv Pharm Technol Res. 2012;4(4):2268-2284.

25. Lv Q, Yu A, Xi Y, et al. Development and evaluation of penciclovirloaded solid lipid nanoparticles for topical delivery. Int $J$ Pharm. 2009;372(1-2):191-198.

26. Kovacevic A, Savic S, Vuleta G, et al. Polyhydroxy surfactants for the formulation of lipid nanoparticles (SLN and NLC): effects on size, physical stability and particle matrix structure. Int J Pharm. 2011;406(1-2):163-172

27. Maryam G, Shohreh F, Alireza V, et.al. Loading hydrophilic drug in solid lipid media as nanoparticles: Statistical modeling of entrapment efficiency and particle size. International Journal of Pharmaceutics. 2012;424(1-2):128-137. 
28. Silva AC, Kumar A, Wild W, et al. Long-term stability, biocompatibility and oral delivery potential of risperidone-loaded solid lipid nanoparticles. Int J Pharm. 2012;436(1-2):798-805.

29. Win HDJ, Paul JB. Drug delivery and nanoparticles: application and hazards. International Journal of nanoparticles. 2008;3(2):133-149.

30. Kunn H, Ajitha S, Wean SC. Lipid-polymer hybrid nanoparticles as a new generation therapeutic delivery platform: A review. European Journal of Pharmaceutics and Biopharmaceutics. 2013;85(3):427-443.

31. Thomas D, Amandine F, Pierre AB, et.al. Encapsulation and release behavior from lipid nanoparticles: model study with nile red fluorophore. Journal of. 2012.
32. Colloid Science and Biotechnology 1: 16-25

33. Gerrits CJ, de Jonge MJ, Schellens JH, et al. Topoisomerase I inhibitors: the relevance of prolonged exposure for present clinical development. Br J Cancer. 1997;76(7):952-962.

34. Dwakanasth BS, Khaitan D, Mathur R. Inhibitors of topoisomerases as anticancer drug: problems and prospects. Indian J Exp Biol. 2004;42(7):649-659. 